\title{
Detection, Occurrence, and Fate of Fluorotelomer Alcohols in Municipal Wastewater Treatment Plants
}

\author{
Hongrui Chen, ${ }^{\dagger, \|}$ Hui Peng, ${ }^{\S} \odot$ Min Yang, ${ }^{\ddagger}, \|_{\odot}$ Jianying $\mathrm{Hu}^{\S}{ }^{\S}$ and Yu Zhang ${ }^{*},, \|_{\odot}$ \\ ${ }^{\dagger}$ Key Laboratory of Drinking Water Science and Technology, Research Center for Eco-Environmental Sciences and ${ }^{\ddagger}$ State Key \\ Laboratory of Environmental Aquatic Chemistry, Research Center for Eco-Environmental Sciences, Chinese Academy of Sciences, \\ Beijing 100085, China \\ ${ }^{\S}$ Laboratory for Earth Surface Processes, College of Urban and Environmental Sciences, Peking University, Beijing 100871, China \\ "University of Chinese Academy of Sciences, Beijing 100049, China
}

Supporting Information

ABSTRACT: Fluorotelomer alcohols (FTOHs) are the most well-known precursors of perfluoroalkyl carboxylic acids (PFCAs), but limited information is available on their occurrence and fate in municipal wastewater treatment plants (WWTPs). The occurrence of FTOHs was investigated in influent, secondary effluent, and sludge of 12 municipal WWTPs in nine cities of China. FTOHs were detected in all WWTPs, and 8:2 FTOH was the predominant congener, with concentrations of $2.10-11.0 \mathrm{ng} / \mathrm{L}, 3.05-12.4 \mathrm{ng} / \mathrm{L}$, and $0.36-$ $1.91 \mathrm{ng} / \mathrm{g}$ dry weight in the influent, secondary effluent, and sludge, respectively. Relatively high proportions of long-chain FTOHs $\left(\mathrm{C}_{10-16}\right)$ were mainly detected in sludge samples. The mass balance of FTOHs and PFCAs in one of the WWTPs with an anaerobic-anoxic-oxic process was further explored. The decrease of mass loads was observed for 4:2 FTOH (mass change percentage: $21 \pm 3.3 \%$ ), 8:2 FTOH (22 $\pm 1.5 \%)$, and 10:2 FTOH $(29 \pm 7.3 \%)$ through aerobic treatment, while the increase of mass loads was observed for 12 PFCAs from $18 \pm 16 \%$ (perfluorononanoic acid) to $165 \pm 15 \%$ (perfluorobutyric acid)), suggesting the potential biotransformation of FTOHs to PFCAs in the aerobic unit. This work provides the first report on the occurrence of FTOHs in sludge samples of municipal WWTPs and their mass balance and highlights a new emission route to environment via WWTPs.

\section{INTRODUCTION}

Fluorotelomer alcohols (FTOHs) are a class of important industrial intermediates used in the synthesis of fluorinated surfactants and polymeric materials and have been applied in a wide range of products such as paints, adhesives, waxes, polishes, metals, electronics, and caulks. ${ }^{1}$ It has been reported the production volume of fluorotelomer increased substantially after the phase-out of PFOS and related products around 2000. ${ }^{2}$ There is increasing evidence that FTOHs can cause endocrine disruptive effects in fish and human cells ${ }^{3-5}$ and reproduction impairment in fish and rat. ${ }^{3,6}$ In addition, FTOHs have been reported to be important precursors of perfluoroalkyl carboxylic acids (PFCAs), and transformation of FTOHs to PFCAs has been documented in different environments including activated sludge, ${ }^{7}$ rats, ${ }^{8}$ humans, ${ }^{9,10}$ and the atmosphere. ${ }^{11}$ Previous studies have suggested that FTOHs might contribute to the increasing temporal trends of longchain PFCAs $(C \geq 8)$ in human serum ${ }^{10}$ and eggs of gulls. ${ }^{12}$

Previous studies have confirmed the global distribution of FTOHs in the air of Europe (181-288 pg/m $\left.\mathrm{m}^{3}\right),{ }^{9,13}$ North America $\left(11-165 \mathrm{pg} / \mathrm{m}^{3}\right){ }^{14}$ and Asia $\left(51.4-1210 \mathrm{pg} / \mathrm{m}^{3}\right){ }^{15}$ The first comprehensive investigation on FTOHs in water environment was conducted in 2008 in Osaka and Kyoto, Japan, and FTOHs were detected in rainwater (1.43-4.07 ng/
$\mathrm{L})$, river water $(<0.13-3.46 \mathrm{ng} / \mathrm{L})$, and the effluents of wastewater treatment plants (WWTPs) $(<0.13-6.67 \mathrm{ng} / \mathrm{L}){ }^{16}$ A recent study has investigated the mass flows and fates of perand polyfluoroalkyl substances in the WWTP of a fluorochemical manufacturing facility, ${ }^{17}$ and the greatest mass flows were observed for 8:2 FTOH (1.5-166 g/d) and 10:2 FTOH (1.7$71 \mathrm{~g} / \mathrm{d}$ ). In spite of the detection of high concentrations (43.8$487 \mu \mathrm{g} / \mathrm{L})$ of FTOHs in the influents of a European industrial WWTP, no target analytes were detected in the corresponding effluent. ${ }^{18}$ Given the fact that FTOHs are widely used for the production of consumer products, domestic wastewater discharge could be considered as one of their routes into the environment. However, the occurrence and fate of FTOHs in municipal WWTPs, particularly for sludge phase, has not yet been studied, partly due to the lack of sensitive analytical method.

In addition, significant increases in PFCA mass flows during activated sludge treatment has become a growing issue in WWTPs. ${ }^{19-23}$ Biodegradation of FTOHs to PFCAs during the

Received: January 17, 2017

Revised: July 13, 2017

Accepted: July 21, 2017

Published: July 21, 2017 
treatment process has been proposed as a potential contributor to the increase of PFCAs in WWTPs. ${ }^{20-22}$ Microbial biodegradation from FTOHs to PFCAs has been well demonstrated in laboratory studies. For example, biotransformation of 8:2 FTOH to perfluorooctanoic acid (PFOA) has been observed in diluted activated sludge after 28 -d incubation $^{24}$ and also in anaerobic digester sludge under methanogenic conditions. ${ }^{25}$ Biotransformation of $6: 2 \mathrm{FTOH}$ to perfluorohexanoic acid (PFHxA) and perfluoropentanoic acid (PFPeA) has also been documented in diluted activated sludge $^{26}$ or anaerobic digester sludge. ${ }^{25}$ While previous studies have highlighted the potential transformation of FTOHs to PFCAs on laboratory scale, the field evidence in municipal WWTPs is still not available.

In this study, the occurrence of six FTOHs (4:2, 6:2, 8:2, $10: 2,12: 2$, and 14:2 FTOH) in 12 municipal WWTPs of China and the behaviors of FTOHs and PFCAs over the course of the wastewater treatment process in a municipal WWTP in Wuxi City with an anaerobic-anoxic-oxic process (A/A/O; Plant A) were explored by mass balance analysis. Among them, due to the lack of standards of 12:2 FTOH and 14:2 FTOH, the two FTOH derivatives were semiquantified as shown in previous studies. $^{27,28}$ In order to increase the analytical sensitivity, the ultra performance liquid chromatography-electrospray ionization-tandem mass spectrometry (UPLC-ESI-MS/MS) method based on dansylation ${ }^{28,29}$ was applied for the simultaneous determination of FTOHs in wastewater and sludge samples from municipal WWTPs. This study will improve our understanding on the fates and emission of FTOHs in municipal WWTPs.

\section{MATERIALS AND METHODS}

Chemicals and Reagents. The four target FTOHs 4:2 FTOH, 6:2 FTOH, 8:2 FTOH, and 10:2 FTOH and four isotope-labeled standards $\left(\mathrm{D}_{4}-4: 2 \mathrm{FTOH},{ }^{13} \mathrm{C}_{2} \mathrm{D}_{2}-6: 2 \mathrm{FTOH}\right.$, ${ }^{13} \mathrm{C}_{2} \mathrm{D}_{2}-8: 2 \mathrm{FTOH}$, and $\left.{ }^{13} \mathrm{C}_{2} \mathrm{D}_{2}-10: 2 \mathrm{FTOH}\right)$ were purchased from Wellington Laboratories Inc. (Guelph, Ontario, Canada). Formic acid (FA), dansyl chloride (DNS), and 4(dimethylamino)pyridine (DMAP) were obtained from Sigma-Aldrich (St. Louis, MO, USA). HPLC-grade methanol $(\mathrm{MeOH})$, acetonitrile $(\mathrm{ACN})$, hexane, and dichloromethane (DCM) were purchased from Fisher Chemicals (New Jersey, USA). Oasis HLB (500 mg, $6 \mathrm{cc}$ ), Oasis WAX (60 mg, $3 \mathrm{cc}$ ) and Sep-Pak silica ( $1 \mathrm{~g}, 6 \mathrm{cc}$ ) solid-phase extraction (SPE) cartridges were purchased from Waters (Milford, MA, USA). Ultrapure water obtained by a Milli-Q RC Synthesis water purification system (Millipore, Bedford, MA, USA) was used in the experiments. Detailed information for chemicals and reagents for perfluoroalkyl acids (PFAAs) (including 12 PFCAs and five perfluoroalkyl sulfonic acids (PFSAs)) analysis is described in the Supporting Information.

Sample Collection. Wastewater and sludge samples were collected from 12 municipal WWTPs (Plant A-Plant L) in nine cities of China from June 2014 to April 2016, with the detailed operational parameters of 12 municipal WWTPs and characteristics of wastewater and sludge in 12 municipal WWTPs shown in Table S1 and Table S2, respectively. Influent, secondary effluent, and return sludge samples were collected from Plant B-Plant L. Mass flow samples were collected from Plant A (anaerobic-anoxic-oxic, A/A/O) in Wuxi City, Jiangsu Province on 6, 7, and 8 June, 2014. Plant A serves about 820,000 inhabitants and treats about $100,000 \mathrm{~m}^{3}$ per day of mainly domestic wastewater. The treatment processes in Plant A comprise screens, a horizontal-flow grit chamber, an upgraded A/A/O with suspended fillers in the latter half of the aerobic tank, and a secondary clarifier. The secondary effluent is further treated with rotary fiber disc filters (RFDFs), which is then designated as final effluent, before discharge to a receiving river. The characteristics of wastewater and sludge in Plant A are presented in Table S3. Another 11 municipal WWTPs (Plant B-Plant L) were located in nine cities of China with the treatment capacity $60,000-2,000,000$ $\mathrm{m}^{3} / \mathrm{d}$ and included three treatment processes $(\mathrm{A} / \mathrm{A} / \mathrm{O}$, oxidation ditch (OD), and cyclic activated sludge technology (CAST)).

For all of the municipal WWTPs, no rain event had taken place in the previous week of the campaign or during sampling days. Flow-proportional $(24 \mathrm{~h})$ composite samples were collected using automatic samplers (SD900, HACH, Loveland, CO, USA), except for the sludge samples, which were collected three times per day and mixed together. The collected samples were stored in $1 \mathrm{~L}$ polypropylene (PP) containers with narrow mouths and screw tops that had been successively washed with $\mathrm{ACN}$, ultrapure water Milli-Q (MQ) water, and then samples from the sampling points. The samples were kept in containers with dry ice and in the dark during the transport from sampling sites to the laboratory. Then the wastewater and sludge samples were centrifuged at the rotational speed of 9,000 rpm (rpm) (J25, Beckman Coulter, USA) for $10 \mathrm{~min}$ to separate wastewater from particulates. The supernatant was vacuum-filtered through glass microfiber filters (GF/F, Whatman, UK) according to previously published protocol, ${ }^{16}$ and then the wastewater samples were stored in the dark at $4{ }^{\circ} \mathrm{C}$ and extracted with SPE cartridges within $24 \mathrm{~h}$ to eliminate potential biodegradation of FTOHs. Sludge was then freeze-dried under vacuum (FD-1A50, Boyikang, China) at low temperature $\left(<-20^{\circ} \mathrm{C}\right)$ to reduce potential biodegradation of FTOHs, homogenized with a mortar and pestle, sieved to obtain the desired particle size (diameter $0.5 \mathrm{~mm}$ ), and stored at $-20{ }^{\circ} \mathrm{C}$, and the extraction was conducted in 1 week. All the samples were collected in triplicate, and extracts were analyzed with UPLC-MS-MS within one month.

Sample Preparation and Dansylation of FTOHs. FTOHs were determined based on a dansylation UPLC-MSMS method as described in recent papers. ${ }^{28,29}$ However, the method has not been validated in wastewater or sludge samples, and therefore we optimized and applied the dansylation method to determine FTOHs in wastewater and sludge samples.

The water-compatible solvent extraction solvents (ACN, $\mathrm{MeOH}, 1: 1 \mathrm{ACN} / \mathrm{MeOH}$ ) were tested for extraction of FTOHs in sludge, and ACN showed the best extraction efficiencies (Figure S1). Thus, ACN was selected as the extraction solvent in the present study. For FTOH analysis in sludge, $0.5 \mathrm{~g} \mathrm{dw}$ of freeze-dried samples spiked with $50 \mu \mathrm{L}$ of $\mathrm{D}_{4}-4: 2$ FTOH $(10 \mu \mathrm{g} / \mathrm{L}),{ }^{13} \mathrm{C}_{2} \mathrm{D}_{2}-6: 2$ FTOH $(10 \mu \mathrm{g} / \mathrm{L})$, ${ }^{13} \mathrm{C}_{2} \mathrm{D}_{2}-8: 2$ FTOH $(10 \mu \mathrm{g} / \mathrm{L})$, and ${ }^{13} \mathrm{C}_{2} \mathrm{D}_{2}-10: 2 \mathrm{FTOH}(10 \mu \mathrm{g} /$ $\mathrm{L})$ was added to $15 \mathrm{~mL}$ PP centrifuge tubes. The samples were left to stand for $24 \mathrm{~h}$ at room temperature in the dark, and then $4 \mathrm{~mL}$ of ACN was added for extraction. After shaking for 20 $\min$ at $300 \mathrm{rpm}$ and sonication for $20 \mathrm{~min}\left(40^{\circ} \mathrm{C}\right)$, the extract was separated by centrifugation at $4000 \mathrm{rpm}$ for $10 \mathrm{~min}$. One milliliter of the extract was diluted with $4 \mathrm{~mL}$ of ultrapure water and then loaded on WAX cartridges which had been conditioned by $3 \mathrm{~mL}$ of $\mathrm{ACN}$ and $3 \mathrm{~mL}$ of ultrapure water. To reduce the elution volume for subsequent dansylation, we 
used small WAX cartridges $(60 \mathrm{mg}, 3 \mathrm{cc}$ ), and $1 \mathrm{~mL}$ of ACN was sufficient to elute the target FTOHs from the cartridges (93-96\% of different FTOHs were eluted). Then, $200 \mu \mathrm{L}$ of DCM containing $30 \mathrm{mg} / \mathrm{mL}$ DNS and $30 \mathrm{mg} / \mathrm{mL}$ DMAP was added to the eluate, which was then shaken vigorously for 1 min. The resulting mixture was kept at $65{ }^{\circ} \mathrm{C}$ for $60 \mathrm{~min}$, and then $3 \mathrm{~mL}$ of ultrapure water and $6 \mathrm{~mL}$ of hexane were added. After $10 \mathrm{~min}$ of shaking at $300 \mathrm{rpm}$, the organic layer was separated by centrifugation at $4000 \mathrm{rpm}$ for $10 \mathrm{~min}$. The extraction process was repeated twice, and the combined extracts were loaded onto silica cartridges conditioned with 8 $\mathrm{mL}$ of DCM and $8 \mathrm{~mL}$ of hexane. The dansylated FTOHs were eluted with $8 \mathrm{~mL}$ of hexane:DCM (v/v, 1:1), blown to dryness, and then dissolved in $0.2 \mathrm{~mL}$ of ACN for UPLC-MS/MS analysis.

FTOHs in wastewater samples $(250 \mathrm{~mL})$ were spiked with $50 \mu \mathrm{L}$ of $\mathrm{D}_{4}-4: 2$ FTOH $(100 \mu \mathrm{g} / \mathrm{L}),{ }^{13} \mathrm{C}_{2} \mathrm{D}_{2}-6: 2$ FTOH $(100$ $\mu \mathrm{g} / \mathrm{L}),{ }^{13} \mathrm{C}_{2} \mathrm{D}_{2}-8: 2$ FTOH $(100 \mu \mathrm{g} / \mathrm{L})$, and ${ }^{13} \mathrm{C}_{2} \mathrm{D}_{2}-10: 2$ FTOH $(100 \mu \mathrm{g} / \mathrm{L})$. The water samples were passed through HLB cartridges which had been conditioned by $8 \mathrm{~mL}$ of ACN and 8 $\mathrm{mL}$ of ultrapure water at a flow rate of $1-2$ drops/s. After the cartridges were dried under a flow of nitrogen, $5 \mathrm{~mL}$ of ACN was used to elute the analytes from the HLB cartridges. We used $0.5 \mathrm{~mL}$ of eluate for dansylation of FTOHs by adding 200 $\mu \mathrm{L}$ of DCM containing $30 \mathrm{mg} / \mathrm{mL}$ of DNS and $30 \mathrm{mg} / \mathrm{mL}$ of DMAP and $300 \mu \mathrm{L}$ of ACN. The reaction solutions were cleaned up using the same strategy as the sludge samples, as mentioned above. Detailed sample preparation for PFAAs analysis is described in the Supporting Information.

UPLC-ESI-MS/MS Analysis. The UPLC apparatus was an Acquity Ultra Performance Liquid Chromatography (Waters, Milford, MA, U.S.). Separation of dansylated FTOHs was conducted using a Waters ACQUITY UPLC BEH phenyl column $(1.7 \mu \mathrm{m} ; 2.1 \mathrm{~mm} \times 100 \mathrm{~mm})$. The column was maintained at $40{ }^{\circ} \mathrm{C}$, and the flow rate and injection volume were $0.3 \mathrm{~mL} / \mathrm{min}$ and $10 \mu \mathrm{L}$, respectively. Methanol (A) and ultrapure water containing $0.1 \%$ formic acid (B) were used as mobile phases. The initial composition of $20 \%$ A was increased to $80 \%$ in $1 \mathrm{~min}$, then increased to $100 \%$ at $5 \mathrm{~min}$, maintained for $2 \mathrm{~min}$, and followed by a decrease to $20 \%$ A, which was then held for $2 \mathrm{~min}$ to allow for equilibration. Mass spectrometry was performed using a Waters Micromass XEVO TQ MS (triple-quadrupole) detector operated with an electrospray ionization source (Micromass, Manchester, UK) in a positive ion mode. The optimized parameters were as follows: source temperature, $150{ }^{\circ} \mathrm{C}$; desolvation temperature, $350{ }^{\circ} \mathrm{C}$; capillary voltage, $3.00 \mathrm{kV}$; cone voltage, $30 \mathrm{~V}$; desolvation gas flow, $800 \mathrm{~L} / \mathrm{h}$; cone gas flow, $50 \mathrm{~L} / \mathrm{h}$. Finally, data were acquired by multiple-reaction monitoring (MRM), and timesegmented scanning was used based on the chromatographic separation of target compounds to maximize detection sensitivity. The MRM transitions and mass spectrometry parameters of FTOHs derivatives and PFAAs in the triple quadrupole instrument are summarized in Table S4. Detailed UPLC-ESI-MS/MS analysis for PFAAs is described in the Supporting Information.

Quantification and Quality Assurance/Quality Control. Identification of the FTOHs was accomplished by comparing the retention time (within 2\%) and the ratio (within 20\%) of the two selected MRM ion transitions with those of standards. To automatically correct for analyte losses during extraction or sample preparation and to compensate for instrument response variations from injection to injection, surrogate standards were used in this study. Concentrations of 4:2 FTOH, 6:2 FTOH, 8:2 FTOH, and 10:2 FTOH in wastewater and sludge samples were corrected using surrogate standards $\mathrm{D}_{4}-4: 2$ FTOH, ${ }^{13} \mathrm{C}_{2} \mathrm{D}_{2}-6: 2$ FTOH, ${ }^{13} \mathrm{C}_{2} \mathrm{D}_{2}-8: 2$ FTOH, and ${ }^{13} \mathrm{C}_{2} \mathrm{D}_{2}-10: 2 \mathrm{FTOH}$, respectively. Due to the lack of standards of 12:2 FTOH and 14:2 FTOH, the two FTOH derivatives were semiquantified using the standard curve of the 10:2 FTOH derivative, as in previous studies, ${ }^{27,28}$ and ${ }^{13} \mathrm{C}_{2} \mathrm{D}_{2}$ 10:2 FTOH was used as the surrogate standard. All equipment rinses were carried out with $\mathrm{ACN}$ to avoid sample contamination. One distilled water blank was incorporated in the analytical procedures for every batch of samples, but no background contamination was found. Recoveries of FTOHs in wastewater and sludge samples were calculated to assess the accuracy of the method, and the relative standard deviation (RSD) was used to evaluate precision. Since it is impossible to obtain sludge or wastewater samples free of analytes, recoveries $(n=5)$ were calculated by subtracting background concentrations in nonspiked samples from spiked samples. The spiked concentrations were 3-6 times higher than the background concentrations in samples, $50 \mathrm{ng} / \mathrm{L}$ for FTOHs in wastewater samples, and $20 \mathrm{ng} / \mathrm{g} \mathrm{dw}$ for FTOHs in return sludge samples. Estimation of the limits of detection (LODs) were based on peak-to-peak noise of the baseline near the analyte peak obtained by analyzing field samples and on a minimal value of signal-to-noise of 3 , while the limits of quantitation (LOQs) were signal-to-noise of 10 . The extent of signal suppression and enhancement in UPLC-ESI-MS/MS was evaluated by spiking standards of dansylated FTOHs into the extracts of wastewater and sludge samples from SPE $(n=5)$, with an analyte addition at least three times the original concentration in samples. The matrix effect observed with each analyte was calculated using the percentage of signal intensity in the sample matrix versus the signal of the same concentration in pure solvent (ACN). Detailed Quantification and Quality Control for PFAAs analysis is described in the Supporting Information.

Mass Balance Calculation. Mass balances were estimated by multiplying concentrations of each analyte by average daily flow rates according to the following equation

$$
W=C_{\text {dissolved }} \times Q+C_{\text {adsorbed }} \times Q \times C_{\text {MLSS }}
$$

where $W$ is the total mass of one target analyte in the aqueous phase and adsorbed to sludge; $C_{\text {dissolved }}$ and $C_{\text {adsorbed }}$ represent the dissolved and adsorbed concentrations, respectively; $Q$ is water flow; and $C_{\text {MLSS }}$ represents the concentration of mixed liquid suspended solids.

The mass change percentage (removal efficiency) for each analyte in each treatment unit was calculated as ( $W_{\text {Inflow }}-$ $\left.W_{\text {Outflow }}\right) / W_{\text {Inflow }} \times 100 \%$, where $W_{\text {Inflow }}$ and $W_{\text {Outflow }}$ represent the mass load of an analyte in inflow and outflow, respectively.

The amounts of FTOHs released daily from the municipal WWTPs were calculated according to the following equation

$$
\text { Mass }=\left(C_{\text {water }} \times Q_{\text {water }}+C_{\text {sludge }} \times Q_{\text {sludge }}\right) \times 10^{-6}
$$

where Mass is the mass loading of an analyte $(\mathrm{g} / \mathrm{d}) ; C_{\text {water }}(\mathrm{ng} /$ $\mathrm{L})$ and $C_{\text {sludge }}(\mathrm{ng} / \mathrm{g} \mathrm{dw})$ are the concentrations of an analyte in the effluent and sludge, respectively; $Q_{\text {water }}\left(\mathrm{m}^{3} / \mathrm{d}\right)$ is the daily flow of effluent; and $Q_{\text {sludge }}(\mathrm{kg} / \mathrm{d})$ is the daily flow of excess sludge. 


\section{DATA ANALYSIS}

Concentrations less than LODs were reported as less than respective $\mathrm{LOD}(<\mathrm{LOD})$. For the calculation, the concentrations less than LODs were treated as zero, and values above the LODs but less than LOQs were assigned a value of half of the respective LOQ. Significance tests were conducted by $t$ test. $P$ values $<0.05$ were regarded as significant.

\section{RESULTS AND DISCUSSION}

Method Performance for Analyzing FTOHs and PFAAs in Wastewater and Sludge Samples. All FTOHs were detected, and the typical MRM chromatograms of FTOHs in sludge and secondary effluent after dansylation and cleanup are presented in Figure S2.

Recoveries of 4:2, 6:2, 8:2, and 10:2 FTOH were $84-112 \%$, $83-116 \%$, and $91-103 \%$ in influent, secondary effluent, and sludge samples, with RSDs less than 8.3\% (Table S5). Besides the recoveries, method sensitivity and matrix effects are also important issues. Background contamination was not found, and the LODs for FTOHs in the influent, secondary effluent, and sludge were in the range of $0.05-0.12 \mathrm{ng} / \mathrm{L}, 0.03-0.11 \mathrm{ng} /$ $\mathrm{L}$, and $0.01-0.07 \mathrm{ng} / \mathrm{g} \mathrm{dw}$, respectively (Table S5). The LODs of FTOHs in wastewater and sludge were 100-1000-fold lower than LOQs in wastewater $(50-100 \mathrm{ng} / \mathrm{L})$ and sludge $(50-100$ $\mathrm{ng} / \mathrm{g} \mathrm{dw}$ ) of recent methods by GC/MS, ${ }^{17}$ highlighting the application potential of the present method for determination of low concentrations of FTOHs in environmental samples.

The potential signal suppression and enhancement of FTOHs in UPLC-ESI-MS/MS detection was also evaluated. The matrix effect for all target analytes was minor, with -5.9 to $14 \%$ in influent, -3.2 to $9.9 \%$ in secondary effluent, and -8.4 to 9.1\% in sludge (Table S5). The improvement in matrix effects was particularly important for the analysis of FTOHs, since signal suppression is a general problem for FTOH analysis using LC-ESI-MS/MS without dansylation, due to their low efficiency in ionization. ${ }^{30}$

Recoveries of PFAAs were $73-109 \%, 72-106 \%$, and $72-$ $105 \%$ in influent, secondary effluent, and sludge samples (Table S6), with RSDs less than $12 \%$. LODs for PFAAs in the influent, secondary effluent, and sludge were in the range of $0.06-0.23$ $\mathrm{ng} / \mathrm{L}, 0.03-0.18 \mathrm{ng} / \mathrm{L}$, and $0.05-0.22 \mathrm{ng} / \mathrm{g} \mathrm{dw}$, respectively (Table S6).

Occurrence and Profiles of FTOHs in 12 Municipal WWTPs. Five among the six FTOHs (exception of 14:2 FTOH) were detected in the wastewater samples of the 12 municipal WWTPs, with total concentrations of four FTOHs ( $\Sigma$ FTOHs, 4:2 FTOH-10:2 FTOH) ranging from 3.78 to 15.1 $\mathrm{ng} / \mathrm{L}$ in the influent and $3.70-17.9 \mathrm{ng} / \mathrm{L}$ in the secondary effluent. 8:2 FTOH was the predominant FTOH detected in all of the wastewater samples $(2.10-12.4 \mathrm{ng} / \mathrm{L})$ with a detection frequency of $100 \%$, followed by 10:2 FTOH $(<0.03-4.63 \mathrm{ng} /$ L) with a detection frequency of $79 \%$ in the influent and $73 \%$ in the secondary effluent (Figure 1(a), Figure S3, and Table 1). The domination of 8:2 FTOH in wastewater was similar to that in air over a WWTP in Tianjin, ${ }^{31}$ but the profile of FTOHs was different from those in air samples over a WWTP in Ontario, in which 6:2 FTOH was the predominant FTOH. ${ }^{32}$ This should be partly due to the different emission profiles of FTOHs between Tianjin and Ontario. On the other hand, the high concentrations of 6:2 FTOH in air samples relative to the other FTOHs should be partly attributed to the higher vapor pressure of $6: 2 \mathrm{FTOH}(876 \pm 159 \mathrm{~Pa})$ than $8: 2 \mathrm{FTOH}(227 \pm 35 \mathrm{~Pa})$

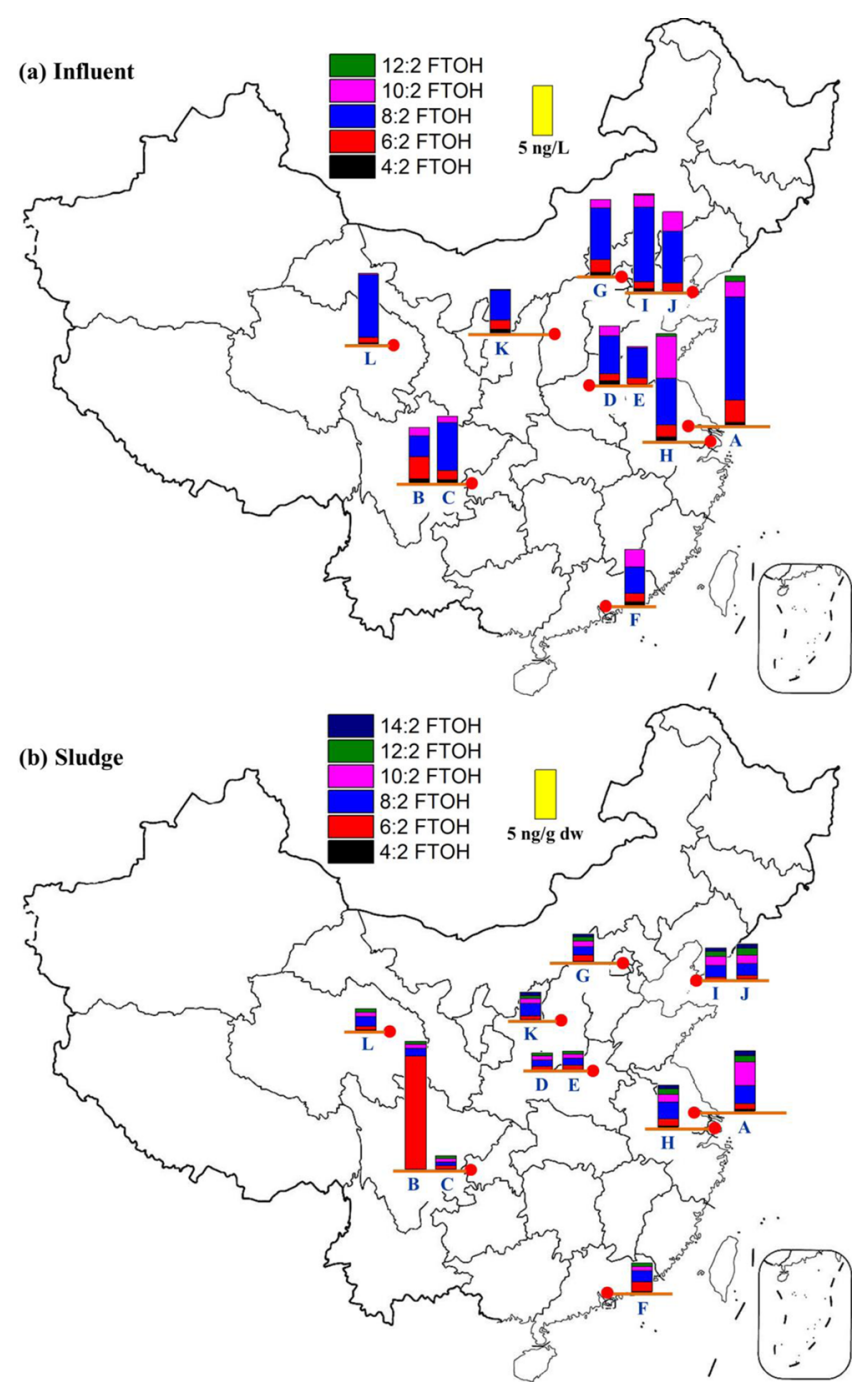

Figure 1. Concentrations and distributions of FTOHs in influent (a) and sludge (b) samples from 12 municipal WWTPs. Due to the lack of standards of 12:2 FTOH and 14:2 FTOH, the two FTOH derivatives were semiquantified.

and 10:2 FTOH $(53 \pm 7 \mathrm{~Pa})$ and thus lead to preference partition of 6:2 FTOH from water to air. ${ }^{33}$ One study has reported the occurrence of $8: 2 \mathrm{FTOH}$ and 10:2 FTOH in WWTP effluents in Japan, and the concentrations $(<0.2-3.24$ $\mathrm{ng} / \mathrm{L}$ for 8:2 FTOH and $<0.2-4.06 \mathrm{ng} / \mathrm{L}$ for 10:2 FTOH) were comparable to the present study. ${ }^{16}$ Another study investigated the occurrence of FTOHs in an effluent sample from a municipal WWTP and an influent sample as well as the corresponding effluent sample from an industrial WWTP. It was found that 6:2 FTOH, 8:2 FTOH, and 10:2 FTOH were only detected in industrial influent with high average concentrations at $487,84.4$, and $43.8 \mu \mathrm{g} / \mathrm{L}$, respectively, ${ }^{98}$ which were much higher than municipal wastewater samples investigated in the present study. A very recent study reported the concentrations of FTOHs in wastewater and sludge samples in an industrial wastewater treatment plant of a fluorochemical manufacturing facility; ${ }^{17}$ but the wastewater treatment plant investigated in their study was not a common situation, and the concentrations of FTOHs were very high and chemical profiles might be very different from common municipal WWTPs. The difference in $\Sigma$ FTOHs concentrations between the influents and secondary effluents was not significant $(p=0.45)$. Except 
Table 1. Concentrations of FTOHs in Influent, Secondary Effluent, and Sludge from 12 Municipal WWTPs in China

\begin{tabular}{|c|c|c|c|c|c|c|c|c|c|c|c|c|}
\hline & \multicolumn{4}{|c|}{ influent $(\mathrm{ng} / \mathrm{L}, n=14)$} & \multicolumn{4}{|c|}{ secondary effluent (ng/L, $n=15)$} & \multicolumn{4}{|c|}{ sludge $(\mathrm{ng} / \mathrm{g}, n=14)$} \\
\hline & range & mean & median & freq $(\%)$ & range & mean & median & $\begin{array}{l}\text { freq } \\
(\%)\end{array}$ & range & mean & median & $\begin{array}{l}\text { freq } \\
(\%)\end{array}$ \\
\hline 4:2 FTOH & $<0.12^{a}-0.40$ & 0.29 & 0.34 & 79 & $0.15-0.35$ & 0.29 & 0.32 & 80 & $0.01-0.20$ & 0.07 & 0.05 & 100 \\
\hline 6:2 FTOH & $0.56-2.28$ & 1.26 & 0.90 & 100 & $0.49-0.98$ & 0.64 & 0.56 & 100 & $0.27-11.4$ & 1.29 & 0.51 & 100 \\
\hline $8: 2 \mathrm{FTOH}$ & $2.10-11.0$ & 5.72 & 5.09 & 100 & $3.05-12.4$ & 5.27 & 3.79 & 100 & $0.36-1.91$ & 1.18 & 1.16 & 100 \\
\hline 10:2 FTOH & $0.12-4.23$ & 1.24 & 1.12 & 79 & $<0.03-4.63$ & 1.07 & 0.57 & 73 & $0.32-2.40$ & 0.92 & 0.50 & 100 \\
\hline$\Sigma \mathrm{FTOHs}^{b}$ & $3.78-15.1$ & 8.51 & 7.45 & $\mathrm{NA}^{c}$ & $3.70-17.9$ & 7.28 & 5.77 & NA & $1.07-12.7$ & 3.47 & 2.44 & NA \\
\hline 12:2 FTOH & $<0.06-0.80$ & 0.15 & $<0.06$ & 36 & $<0.05-0.34$ & 0.02 & $<0.05$ & 6.7 & $0.28-0.85$ & 0.45 & 0.38 & 100 \\
\hline 14:2 FTOH & $<0.09$ & $<0.09$ & $<0.09$ & 0 & $<0.08$ & $<0.08$ & $<0.08$ & 0 & $<0.05-0.56$ & 0.24 & 0.32 & 57 \\
\hline
\end{tabular}

$a_{<x}$ : concentrations less than respective LOD. ${ }^{b} \Sigma$ FTOHs: the total concentrations of 4:2 FTOH, 6:2 FTOH, 8:2 FTOH, and 10:2 FTOH. ${ }^{c}$ NA: not available. Due to the lack of standards of 12:2 FTOH and 14:2 FTOH, the two FTOH derivatives were semiquantified.

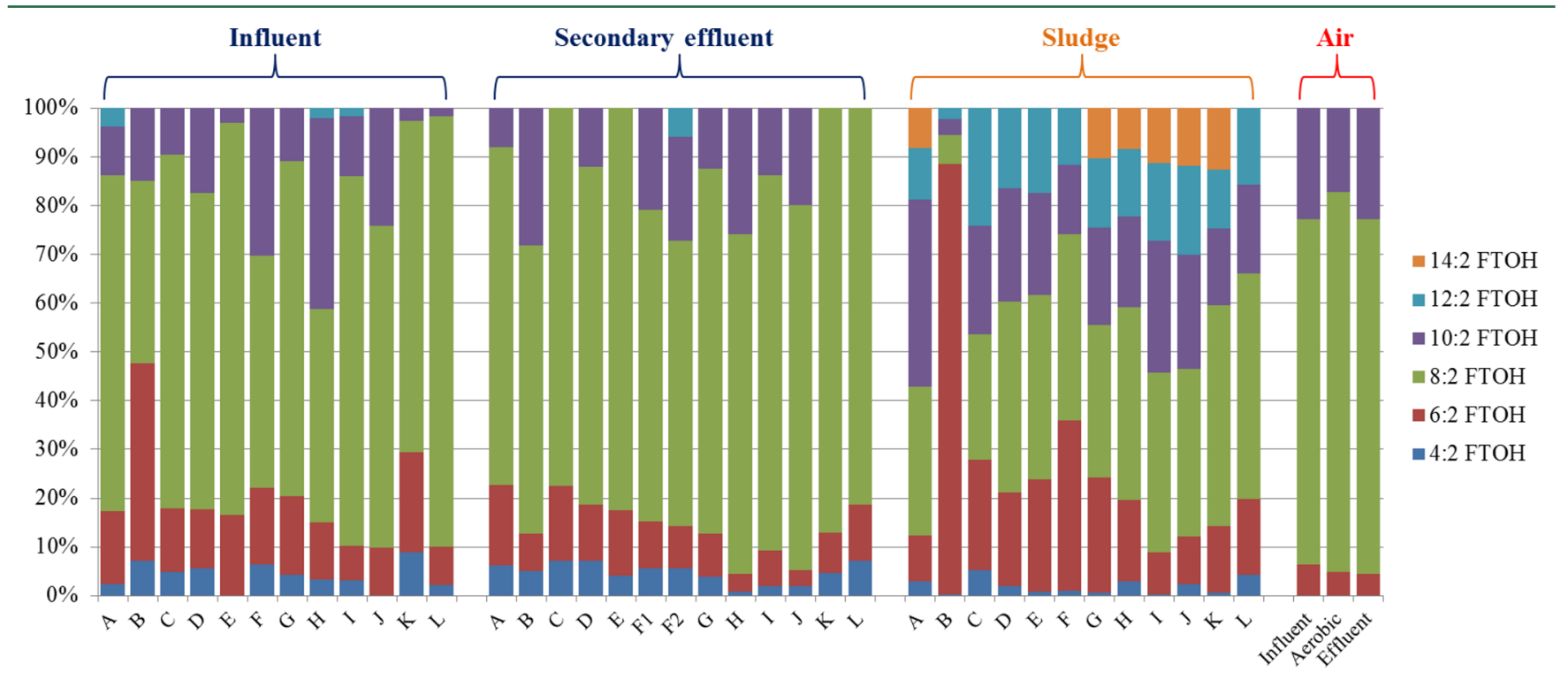

Figure 2. Profiles of FTOHs in wastewater, sludge, and air. The FTOH concentrations in wastewater and sludge were from this study; the FTOH concentrations in air were from ref 31. Due to the lack of standards of 12:2 FTOH and 14:2 FTOH, the two FTOH derivatives were semiquantified.

for 6:2 FTOH, which showed significantly lower concentrations in the secondary effluent $(p=0.01)$, the other FTOHs did not exhibit significant differences between the influents and secondary effluents ( $p$ values: $0.12-0.95$ ). These results indicated that FTOHs could not be efficiently removed by municipal WWTPs.

All FTOHs were detected in the sludge samples from 12 municipal WWTPs, with a detection frequency of $100 \%$ except for 14:2 FTOH (57\%). To our knowledge, only one study reported the occurrence of FTOHs in wastewater samples from the WWTP, ${ }^{16}$ and so this is the first report on the occurrence of FTOHs in sludge of municipal WWTPs, and the concentrations of $\Sigma$ FTOHs in sludge were $1.07-12.7 \mathrm{ng} / \mathrm{g}$ dw. It was similar to wastewater samples; 8:2 FTOH was detected as the predominant congener in sludge samples, with an average concentration of $1.18 \mathrm{ng} / \mathrm{g} \mathrm{dw}$ (Figure $1(\mathrm{~b})$ and Table 1). It should be noted that the concentrations of FTOHs in sludge samples were lower than the LODs (50-100 ng/g $\mathrm{dw}$ ) of recent methods by GC/MS, ${ }^{17}$ showing that the sensitive derivatization method developed in the present study was critical for the determination of FTOHs in environmental matrices with low concentrations. In contrast to the wastewater samples, long-chain FTOHs including 10:2 FTOH (0.32-2.40 $\mathrm{ng} / \mathrm{g} \mathrm{dw}), 12: 2 \mathrm{FTOH}(0.28-0.85 \mathrm{ng} / \mathrm{g} \mathrm{dw})$, and 14:2 FTOH $(<0.05-0.56 \mathrm{ng} / \mathrm{g} \mathrm{dw})$ were detected at relatively high concentrations in the sludge samples (Figure 2). This phenomenon should be partly attributed to the preferential sorption of long-chain FTOHs to sludge from water, as indicated in previous laboratory studies, ${ }^{34}$ that the increase of each $\mathrm{CF}_{2}$ moiety decreased the aqueous solubility by $\sim 0.78 \mathrm{log}$ units. At the same time, in contrast to the occurrence of FTOHs (0.05-0.15 ng/g dw for 8:2 FTOH, 0.08-0.26 ng/g $\mathrm{dw}$ for 10:2 FTOH) in six sediment samples from Liaodong Bay, $^{29}$ 8:2 FTOH $(0.36-1.91 \mathrm{ng} / \mathrm{g} \mathrm{dw})$ and 10:2 FTOH $(0.32-2.40 \mathrm{ng} / \mathrm{g} \mathrm{dw})$ in the present study were detected at relatively high concentrations. The observed preferential partition of the long-chain FTOHs in excess sludge highlighted an environmental risk for the application of dehydrated sludge on agriculture fields, as exemplified by the occurrence of FTOHs (5-73 ng/g dw for 8:2 FTOH, <5.6-166 ng/g dw for 10:2 FTOH, 2-133 ng/g dw for 12:2 FTOH, and 3-52 ng/g $\mathrm{dw}$ for 14:2 FTOH) in sludge-applied soils from the United States. ${ }^{27}$ While the sludge analyzed was heavily polluted by a manufacturer, ${ }^{27}$ combined with the wide occurrence of FTOHs in sludge samples determined in the present study, it was suggested that the potential environmental risk from the municipal WWTPs. Future studies are warranted to more broadly investigate the potential occurrence and risk of FTOHs in sludge-applied soils. 

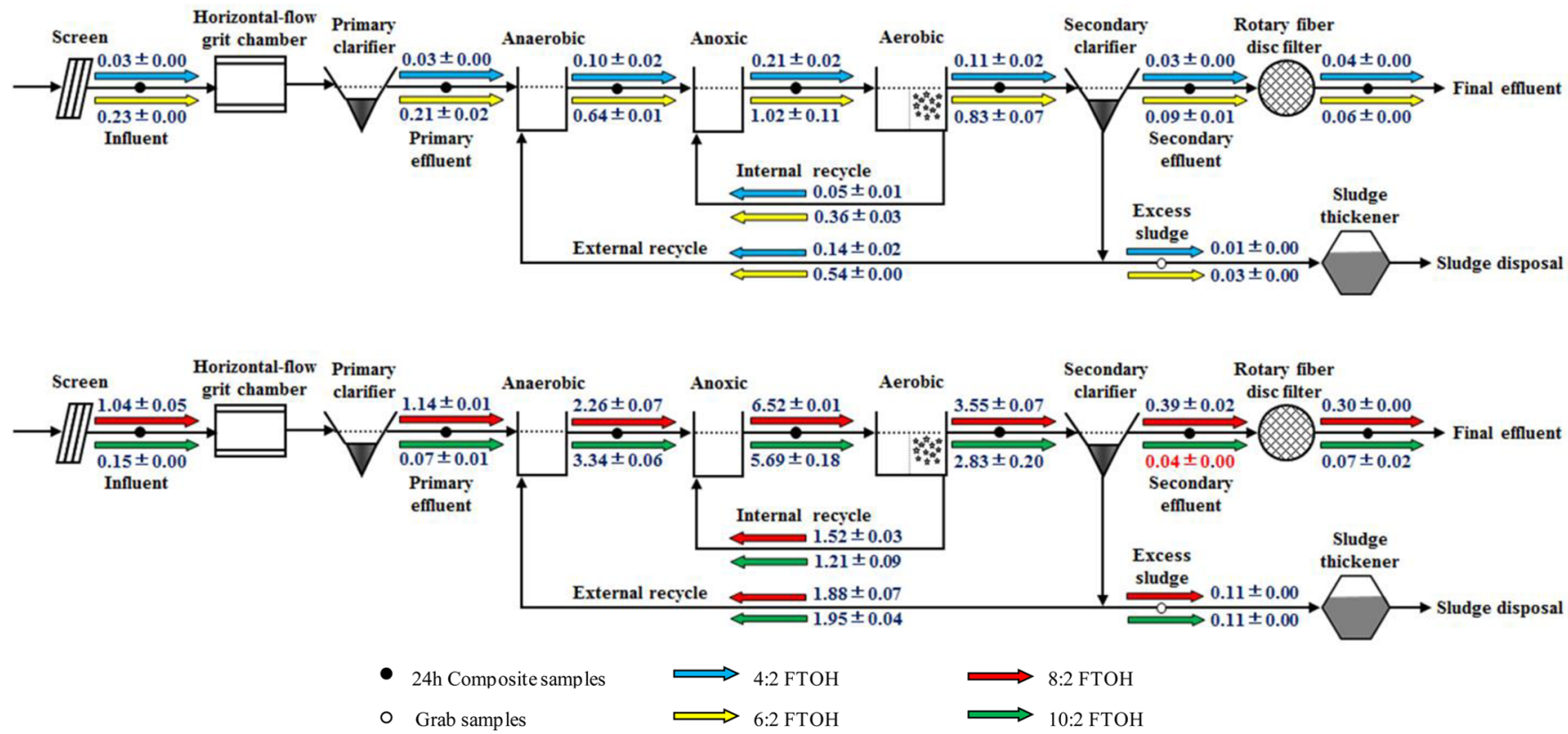

Figure 3. Total mass flow (g/d) of 4:2 FTOH, 6:2 FTOH, 8:2 FTOH, and 10:2 FTOH along the treatment processes in Plant A (A/A/O).

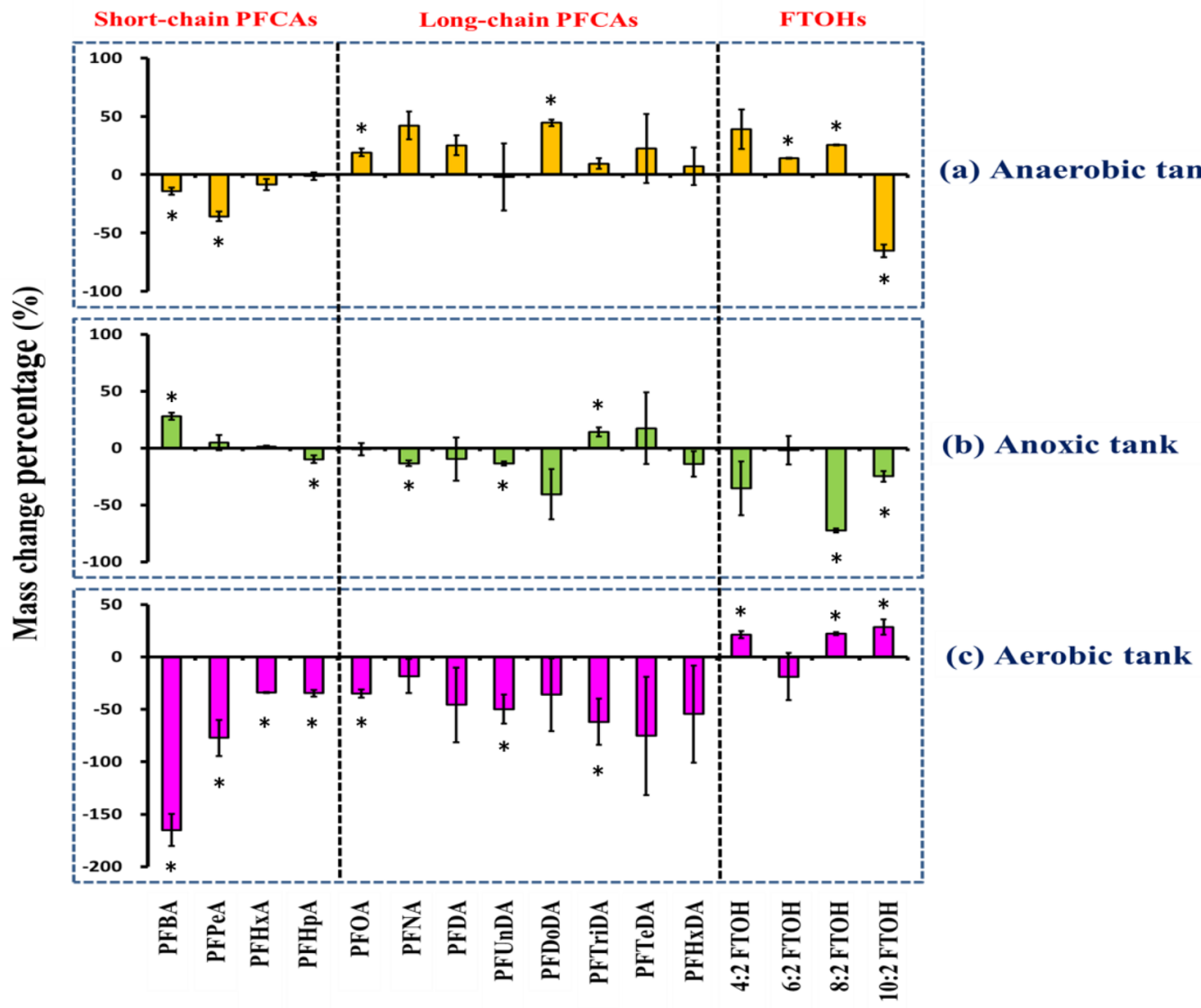

Figure 4. Mass change percentages (removal efficiency) of PFCAs and FTOHs in three treatment processes including anaerobic tank (a), anoxic tank (b), and aerobic tank (c) of Plant A (A/A/O). “*” indicates a statistically significant result.

Behaviors of FTOHs and PFCAs along Wastewater Treatment Process. The concentrations of FTOHs and PFAAs (including 12 PFCAs and 5 PFSAs) over the whole treatment process of Plant $\mathrm{A}(\mathrm{A} / \mathrm{A} / \mathrm{O})$ are presented in Table S7 and Table S8. Generally decreasing concentrations of
FTOHs in wastewater samples were observed along the treatment process. For example, the concentration of $8: 2$ FTOH was $10.4 \pm 0.53 \mathrm{ng} / \mathrm{L}$ in the influent, $3.73 \pm 0.15 \mathrm{ng} / \mathrm{L}$ in the anaerobic wastewater samples, and $2.99 \pm 0.01 \mathrm{ng} / \mathrm{L}$ in the final effluent, indicating part of FTOHs from wastewater 
may be transformed during the treatment. The concentrations of PFCAs were similar between influent and effluent, except for perfluorobutyric acid (PFBA) whose concentrations were increased from $24.8 \pm 3.69 \mathrm{ng} / \mathrm{L}$ to $101 \pm 13.1 \mathrm{ng} / \mathrm{L}$. To better understand the behaviors of FTOHs in municipal WWTPs, we calculated the mass flow (wastewater and sludge) of FTOHs and PFCAs in Plant A (A/A/O) as shown in Figure 3 and Figure S4, as well as the mass change percentages of FTOHs and PFCAs in the three basic treatment units of Plant A, namely the anaerobic, anoxic, and aerobic tanks (Figure 4).

In the anaerobic tank, 4:2 FTOH, 6:2 FTOH, and 8:2 FTOH were found to be increased by $39 \pm 17 \%, 14 \pm 0.43 \%$, and $25 \pm 0.47 \%$, respectively, while 10:2 FTOH decreased significantly by $65 \pm 5.5 \%$. In the anoxic tank, the mass loads of $\Sigma$ FTOHs increased from $9.48 \pm 0.07 \mathrm{~g} / \mathrm{d}$ to $13.4 \pm 0.26 \mathrm{~g} / \mathrm{d}$, with 4:2 FTOH, 6:2 FTOH, 8:2 FTOH, and 10:2 FTOH increased by $36 \pm 24 \%, 1.8 \pm 13 \%, 73 \pm 1.9 \%$, and $25 \pm 4.5 \%$, respectively ( $p<0.05$ for 8:2 FTOH and 10:2 FTOH) (Figure 4(b)), suggesting the presence of some precursors of FTOHs such as polyfluoroalkyl phosphates (PAPs), ${ }^{35}$ fluorotelomer acrylate (FTAC), and fluorotelomer methacrylate (FTMAC). ${ }^{36}$ A previous study confirmed the presence of PAPs in sludge at high concentrations ranging from 47 to $200 \mathrm{ng} / \mathrm{g}$. ${ }^{37}$ Therefore, further study is warranted to clarify the potential occurrence of these upstream precursors in municipal WWTPs and their contribution to FTOH mass loads. In the aerobic tank, the mass loads of $\Sigma$ FTOHs decreased from $13.4 \pm 0.26 \mathrm{~g} / \mathrm{d}$ to 10.5 $\pm 0.32 \mathrm{~g} / \mathrm{d}$, with $4: 2,8: 2$, and 10:2 FTOH decreased by $21 \pm$ $3.3 \%(p=0.01), 22 \pm 1.5 \%(p=0.01)$, and $29 \pm 7.3 \%(p=$ 0.03 ), respectively (Figure $4(c)$ ). This result showed that biodegradation of FTOHs might occur in aerobic tanks, which agreed with previous laboratory biodegradation tests. ${ }^{24,38,39}$ While it cannot be strictly excluded that such loss of FTOHs in the aerobic tank was due to emit into air as reported in previous studies, ${ }^{32,40-42}$ the significant loss of nonvolatile 10:2 FTOH at a similar extent to 4:2 $\mathrm{FTOH}$, and also the specific loss of FTOHs in the aerobic tank, indicated that such mass decrease of FTOHs should be largely attributed to biodegradation.

As for PFCAs, while congener-specific increase or decrease in mass flows were found in the different units, the most pronouncing change was found in the aerobic tank where the mass flows of all 12 PFCAs increased. The mass loads of total PFCAs increased from $94.7 \pm 4.43 \mathrm{~g} / \mathrm{d}$ to $161 \pm 4.23 \mathrm{~g} / \mathrm{d}$ before and after the aerobic tank. The mass change percentages of all PFCAs in aerobic tank were in a range from $18 \pm 16 \%$ (PFNA) to $165 \pm 15 \%$ (PFBA) ( $p<0.05$ for PFBA, PFPeA, PFHxA, PFHpA, PFOA, PFUnDA, and PFTriDA) (Figure 4(c)). Such results were consistent with the significant loss of FTOHs in the same aerobic tank, indicating the potential biotransformation of FTOHs to PFCAs. Such preference biodegradation of FTOHs to PFCAs under aerobic conditions has been documented in previous laboratory studies. ${ }^{24,38,39} \mathrm{It}$ should be noted that the increase of mass loads of PFPeA in aerobic tank was $6.29 \pm 1.30 \mathrm{~g} / \mathrm{d}$, about 100 -fold greater those $(0.04 \pm 0.00 \mathrm{~g} / \mathrm{d})$ of corresponding decreasing mass loads of precursor 4:2 FTOH. Such phenomenon showed that the mass increase of PFCAs could not be completely explained by the biotransformation of FTOHs, and future studies are warranted to clarify the potential biotransformation of other precursors such as PAPs. ${ }^{35,43,44}$ While previous studies have widely highlighted the potential biotransformation of FTOHs to PFCAs in laboratory experiments, ${ }^{24,39,45}$ with the molar yield of PFOA from 8:2 FTOH ranging from $0.5 \%$ in a pure bacterial culture $^{38}$ to $40 \%$ in one aerobic soil, ${ }^{46}$ the present study provided the field evidence for potential biotransformation of FTOHs to PFCAs in the aerobic treatment.

Discharge of FTOHs to Environment. Due to the lack of sludge discharge information on four plants (Plants B, D, E, F) in the 12 investigated municipal WWTPs, daily mass loadings of FTOHs (g/d) to environment from eight investigated municipal WWTPs are presented in Table S9. The discharge of $\Sigma$ FTOHs (4:2 FTOH-10:2 FTOH) from the secondary effluent and sludge was calculated to be $0.30-35.8 \mathrm{~g} / \mathrm{d}$ and 0.02-1.01 g/d, respectively. While aqueous discharge via effluent was the main discharge route for 4:2 FTOH (8199\%), 6:2 FTOH (77-96\%), and 8:2 FTOH (81-99\%), sludge discharge via excess sludge was the main discharge route for 12:2 FTOH (100\%) and 14:2 FTOH (100\%). The discharge of $\Sigma$ FTOHs in effluent and sludge was calculated as $0.05-0.18 \mathrm{~g}$ per $10^{4} \times \mathrm{m}^{3}$ wastewater. ${ }^{41}$ The discharge of excess sludge without further treatment significantly contributes to the environmental burden of FTOHs, and further studies are warranted to effectively eliminate FTOH residuals in sludge.

Overall, this work provides the first report on the occurrence of FTOHs in sludge samples of municipal WWTPs and their mass balance and indicates part of the FTOHs may have been transformed to PFCAs in the aerobic tank. The emission data for FTOHs presented here is useful for justification of future waste sector studies. The decrease of the mass load of total FTOHs and the simultaneous increase of PFCAs in the aerobic tanks suggested the potential biotransformation of FTOHs to PFCAs, while FTOHs increase in the anoxic tank suggested that precursors of FTOHs may exist in wastewater, which will require further clarification.

\section{ASSOCIATED CONTENT}

\section{Supporting Information}

The Supporting Information is available free of charge on the ACS Publications website at DOI: 10.1021/acs.est.7b00315.

Text, figures, and tables of (1) chemicals and reagents, (2) sample preparation, (3) UPLC-ESI-MS/MS, and (4) quantification and quality control for PFAAs analysis; (5) comparison of FTOH concentrations in one same municipal sludge sample; (6) UPLC-MS/MS MRM chromatograms of FTOHs in standards after dansylation; (7) concentrations and distributions of FTOHs in secondary effluent samples from 12 municipal WWTPs; (8) total mass flow of 12 PFCAs along the treatment processes in Plant A (A/A/O); (9) operational parameters of 12 municipal WWTPs; (10) characteristics of wastewater and sludge in 12 municipal WWTPs; (11) characteristics of wastewater and sludge in Plant A (A/A) $\mathrm{O})$; (12) MRM transitions and mass spectrometry parameters of FTOH derivatives and PFAAs in the triple quadrupole instrument; (13) LODs, LOQs, recoveries, and ME of FTOHs in wastewater and sludge; (14) LODs, LOQs, and recoveries of PFAAs in wastewater and sludge; (15) concentrations of FTOHs in wastewater and sludge samples of Plant A (A/A/O); (16) concentrations of PFAAs in wastewater and sludge samples of Plant A (A/A/O); (17) discharge of FTOHs from effluent and sludge of municipal WWTPs to environment (PDF) 


\section{AUTHOR INFORMATION}

\section{Corresponding Author}

*Phone: +86-10-62919883. Fax: +86-10-62923541. E-mail:

zhangyu@rcees.ac.cn.

ORCID ${ }^{\circ}$

Hui Peng: 0000-0002-2777-0588

Min Yang: 0000-0002-9034-3175

Jianying Hu: 0000-0003-1965-7494

Yu Zhang: 0000-0003-1017-4170

\section{Notes}

The authors declare no competing financial interest.

\section{ACKNOWLEDGMENTS}

Financial support for this study was obtained from the Major Science and Technology Program for Water Pollution Control and Treatment (2017ZX07106005). We thank Dr. Xingcan Zheng, Mrs. Wei Shang, and Mr. Kemin Ma for their help with the sample collection.

\section{REFERENCES}

(1) Dinglasan, M. J. A.; Ye, Y.; Edwards, E. A.; Mabury, S. A Fluorotelomer alcohol biodegradation yields poly- and perfluorinated acids. Environ. Sci. Technol. 2004, 38 (10), 2857-2864.

(2) D'Eon, J. C.; Mabury, S. A. Exploring Indirect Sources of Human Exposure to Perfluoroalkyl Carboxylates (PFCAs): Evaluating Uptake, Elimination, and Biotransformation of Polyfluoroalkyl Phosphate Esters (PAPs) in the Rat. Environ. Health Persp. 2011, 119 (3), 344-350.

(3) Liu, C.; Deng, J.; Yu, L.; Ramesh, M.; Zhou, B. Endocrine disruption and reproductive impairment in zebrafish by exposure to 8:2 fluorotelomer alcohol. Aquat. Toxicol. 2010, 96 (1), 70-76.

(4) Ishibashi, H.; Yamauchi, R.; Matsuoka, M.; Kim, J. W.; Hirano, M.; Yamaguchi, A.; Tominaga, N.; Arizono, K. Fluorotelomer alcohols induce hepatic vitellogenin through activation of the estrogen receptor in male medaka (Oryzias latipes). Chemosphere 2008, 71 (10), 18531859.

(5) Maras, M.; Vanparys, C.; Muylle, F.; Robbens, J.; Berger, U.; Barber, J. L.; Blust, R.; De Coen, W. Estrogen-like properties of fluorotelomer alcohols as revealed by MCF-7 breast cancer cell proliferation. Environ. Health Persp. 2006, 114 (1), 100-105.

(6) Mylchreest, E.; Ladics, G. S.; Munley, S. M.; Buck, R. C.; Stadler, J. C. Evaluation of the reproductive and developmental toxicity of a fluoroalkylethanol mixture. Drug Chem. Toxicol. 2005, 28 (2), 159175.

(7) Wang, N.; Szostek, B.; Buck, R. C.; Folsom, P. W.; Sulecki, L. M.; Capka, V.; Berti, W. R.; Gannon, J. T. Fluorotelomer alcohol biodegradation - Direct evidence that perfluorinated carbon chains breakdown. Environ. Sci. Technol. 2005, 39 (19), 7516-7528.

(8) Fasano, W. J.; Carpenter, S. C.; Gannon, S. A.; Snow, T. A.; Stadler, J. C.; Kennedy, G. L.; Buck, R. C.; Korzeniowski, S. H.; Hinderliter, P. M.; Kemper, R. A. Absorption, distribution, metabolism, and elimination of 8-2 fluorotelomer alcohol in the rat. Toxicol. Sci. 2006, 91 (2), 341-355.

(9) Nilsson, H.; Karrman, A.; Rotander, A.; van Bavel, B.; Lindstrom, G.; Westberg, H. Inhalation Exposure to Fluorotelomer Alcohols Yield Perfluorocarboxylates in Human Blood? Environ. Sci. Technol. 2010, 44 (19), 7717-7722.

(10) Gomis, M. I.; Vestergren, R.; Nilsson, H.; Cousins, I. T. Contribution of Direct and Indirect Exposure to Human Serum Concentrations of Perfluorooctanoic Acid in an Occupationally Exposed Group of Ski Waxers. Environ. Sci. Technol. 2016, 50 (13), 7037-7046.

(11) Ellis, D. A.; Martin, J. W.; De Silva, A. O.; Mabury, S. A.; Hurley, M. D.; Andersen, M. P. S.; Wallington, T. J. Degradation of fluorotelomer alcohols: A likely atmospheric source of perfluorinated carboxylic acids. Environ. Sci. Technol. 2004, 38 (12), 3316-3321.
(12) Gewurtz, S. B.; Martin, P. A.; Letcher, R. J.; Burgess, N. M.; Champoux, L.; Elliott, J. E.; Weseloh, D. V. C. Spatio-temporal trends and monitoring design of perfluoroalkyl acids in the eggs of gull (Larid) species from across Canada and parts of the United States. Sci. Total Environ. 2016, 565, 440-450.

(13) Jahnke, A.; Ahrens, L.; Ebinghaus, R.; Temme, C. Urban versus remote air concentrations of fluorotelomer alcohols and other polyfluorinated alkyl substances in Germany. Environ. Sci. Technol. 2007, 41 (3), 745-752.

(14) Stock, N. L.; Lau, F. K.; Ellis, D. A.; Martin, J. W.; Muir, D. C. G.; Mabury, S. A. Polyfluorinated telomer alcohols and sulfonamides in the North American troposphere. Environ. Sci. Technol. 2004, 38 (4), 991-996.

(15) Li, J.; Del Vento, S.; Schuster, J.; Zhang, G.; Chakraborty, P.; Kobara, Y.; Jones, K. C. Perfluorinated Compounds in the Asian Atmosphere. Environ. Sci. Technol. 2011, 45 (17), 7241-7248.

(16) Mahmoud, M. A. M.; Karrman, A.; Oono, S.; Harada, K. H.; Koizumi, A. Polyfluorinated telomers in precipitation and surface water in an urban area of Japan. Chemosphere 2009, 74 (3), 467-472.

(17) Dauchy, X.; Boiteux, V.; Bach, C.; Colin, A.; Hemard, J.; Rosin, C.; Munoz, J. F. Mass flows and fate of per- and polyfluoroalkyl substances (PFASs) in the wastewater treatment plant of a fluorochemical manufacturing facility. Sci. Total Environ. 2017, 576, $549-558$.

(18) Gremmel, C.; Fromel, T.; Knepper, T. P. HPLC-MS/MS methods for the determination of 52 perfluoroalkyl and polyfluoroalkyl substances in aqueous samples. Anal. Bioanal. Chem. 2017, 409 (6), 1643-1655.

(19) Schultz, M. M.; Higgins, C. P.; Huset, C. A.; Luthy, R. G.; Barofsky, D. F.; Field, J. A. Fluorochemical mass flows in a municipal wastewater treatment facility. Environ. Sci. Technol. 2006, 40 (23), $7350-7357$.

(20) Sinclair, E.; Kannan, K. Mass loading and fate of perfluoroalkyl surfactants in wastewater treatment plants. Environ. Sci. Technol. 2006, 40 (5), 1408-1414.

(21) Yu, J.; Hu, J.; Tanaka, S.; Fujii, S. Perfluorooctane sulfonate (PFOS) and perfluorooctanoic acid (PFOA) in sewage treatment plants. Water Res. 2009, 43 (9), 2399-2408.

(22) Kunacheva, C.; Tanaka, S.; Fujii, S.; Boontanon, S. K.; Musirat, C.; Wongwattana, T.; Shivakoti, B. R. Mass flows of perfluorinated compounds (PFCs) in central wastewater treatment plants of industrial zones in Thailand. Chemosphere 2011, 83 (6), 737-744.

(23) Kim, S. K.; Im, J. K.; Kang, Y. M.; Jung, S. Y.; Kho, Y. L.; Zoh, K. D. Wastewater treatment plants (WWTPs)-derived national discharge loads of perfluorinated compounds (PFCs). J. Hazard. Mater. 2012, 201-202, 82-91.

(24) Wang, N.; Szostek, B.; Folsom, P. W.; Sulecki, L. M.; Capka, V.; Buck, R. C.; Berti, W. R.; Gannon, J. T. Aerobic biotransformation of C-14-labeled 8-2 telomer B alcohol by activated sludge from a domestic sewage treatment plant. Environ. Sci. Technol. 2005, 39 (2), $531-538$.

(25) Zhang, S.; Szostek, B.; McCausland, P. K.; Wolstenholme, B. W.; Lu, X.; Wang, N.; Buck, R. C. 6:2 and 8:2 Fluorotelomer Alcohol Anaerobic Biotransformation in Digester Sludge from a WWTP under Methanogenic Conditions. Environ. Sci. Technol. 2013, 47 (9), 42274235.

(26) Liu, J.; Wang, N.; Szostek, B.; Buck, R. C.; Panciroli, P. K.; Folsom, P. W.; Sulecki, L. M.; Bellin, C. A. 6-2 Fluorotelomer alcohol aerobic biodegradation in soil and mixed bacterial culture. Chemosphere 2010, 78 (4), 437-444.

(27) Yoo, H.; Washington, J. W.; Ellington, J. J.; Jenkins, T. M.; Neill, M. P. Concentrations, Distribution, and Persistence of Fluorotelomer Alcohols in Sludge-Applied Soils near Decatur, Alabama, USA. Environ. Sci. Technol. 2010, 44 (22), 8397-8402.

(28) Yuan, G.; Peng, H.; Huang, C.; Hu, J. Ubiquitous Occurrence of Fluorotelomer Alcohols in Eco-Friendly Paper-Made Food-Contact Materials and Their Implication for Human Exposure. Environ. Sci. Technol. 2016, 50 (2), 942-950. 
(29) Peng, H.; Hu, K.; Zhao, F.; Hu, J. Derivatization method for sensitive determination of fluorotelomer alcohols in sediment by liquid chromatography-electrospray tandem mass spectrometry. J. Chromatogr. A 2013, 1288, 48-53.

(30) Chu, S.; Letcher, R. J. Analysis of fluorotelomer alcohols and perfluorinated sulfonamides in biotic samples by liquid chromatography-atmospheric pressure photoionization mass spectrometry. $J$. Chromatogr. A 2008, 1215 (1-2), 92-99.

(31) Yao, Y. M.; Chang, S.; Sun, H. W.; Gan, Z. W.; Hu, H. W.; Zhao, Y. Y.; Zhang, Y. F. Neutral and ionic per- and polyfluoroalkyl substances (PFASs) in atmospheric and dry deposition samples over a source region (Tianjin, China). Environ. Pollut. 2016, 212, 449-456.

(32) Ahrens, L.; Shoeib, M.; Harner, T.; Lee, S. C.; Guo, R.; Reiner, E. J. Wastewater Treatment Plant and Landfills as Sources of Polyfluoroalkyl Compounds to the Atmosphere. Environ. Sci. Technol. 2011, 45 (19), 8098-8105.

(33) Lei, Y. D.; Wania, F.; Mathers, D.; Mabury, S. A. Determination of vapor pressures, octanol-air, and water-air partition coefficients for polyfluorinated sulfonamide, sulfonamidoethanols, and telomer alcohols. J. Chem. Eng. Data 2004, 49 (4), 1013-1022.

(34) Liu, J.; Lee, L. S. Effect of fluorotelomer alcohol chain length on aqueous solubility and sorption by soils. Environ. Sci. Technol. 2007, 41 (15), 5357-5362.

(35) Lee, H.; D'Eon, J.; Mabury, S. A. Biodegradation of Polyfluoroalkyl Phosphates as a Source of Perfluorinated Acids to the Environment. Environ. Sci. Technol. 2010, 44 (9), 3305-3310.

(36) Royer, L. A.; Lee, L. S.; Russell, M. H.; Nies, L. F.; Turco, R. F. Microbial transformation of 8:2 fluorotelomer acrylate and methacrylate in aerobic soils. Chemosphere 2015, 129, 54-61.

(37) D’Eon, J. C.; Crozier, P. W.; Furdui, V. I.; Reiner, E. J.; Libelo, E. L.; Mabury, S. A. Observation of a Commercial Fluorinated Material, the Polyfluoroalkyl Phosphoric Acid Diesters, in Human Sera, Wastewater Treatment Plant Sludge, and Paper Fibers. Environ. Sci. Technol. 2009, 43 (12), 4589-4594.

(38) Liu, J.; Lee, L. S.; Nies, L. F.; Nakatsu, C. H.; Turco, R. F. Biotransformation of 8:2 fluorotelomer alcohol in soil and by soil bacteria isolates. Environ. Sci. Technol. 2007, 41 (23), 8024-8030.

(39) Zhao, L.; McCausland, P. K.; Folsom, P. W.; Wolstenholme, B. W.; Sun, H.; Wang, N.; Buck, R. C. 6:2 Fluorotelomer alcohol aerobic biotransformation in activated sludge from two domestic wastewater treatment plants. Chemosphere 2013, 92 (4), 464-470.

(40) Vierke, L.; Ahrens, L.; Shoeib, M.; Reiner, E. J.; Guo, R.; Palm, W.-U.; Ebinghaus, R.; Harner, T. Air concentrations and particle-gas partitioning of polyfluoroalkyl compounds at a wastewater treatment plant. Environ. Chem. 2011, 8 (4), 363-371.

(41) Weinberg, I.; Dreyer, A.; Ebinghaus, R. Waste water treatment plants as sources of polyfluorinated compounds, polybrominated diphenyl ethers and musk fragrances to ambient air. Environ. Pollut. 2011, 159 (1), 125-132.

(42) Xiao, F.; Halbach, T. R.; Simcik, M. F.; Gulliver, J. S. Input characterization of perfluoroalkyl substances in wastewater treatment plants: Source discrimination by exploratory data analysis. Water Res. 2012, 46 (9), 3101-3109.

(43) D'Eon, J. C.; Mabury, S. A. Production of perfluorinated carboxylic acids (PFCAs) from the biotransformation of polyfluoroalkyl phosphate surfictants (PAPS): Exploring routes of human contamination. Environ. Sci. Technol. 2007, 41 (13), 4799-4805.

(44) Liu, C.; Liu, J. Aerobic biotransformation of polyfluoroalkyl phosphate esters (PAPs) in soil. Environ. Pollut. 2016, 212, 230-237. (45) Zhang, S.; Merino, N.; Wang, N.; Ruan, T.; Lu, X. Impact of 6:2 fluorotelomer alcohol aerobic biotransformation on a sediment microbial community. Sci. Total Environ. 2017, 575, 1361-1368.

(46) Wang, N.; Szostek, B.; Buck, R. C.; Folsom, P. W.; Sulecki, L. M.; Gannon, J. T. 8-2 Fluorotelomer alcohol aerobic soil biodegradation: Pathways, metabolites, and metabolite yields. Chemosphere 2009, 75 (8), 1089-1096. 